\title{
Feasibility study of using montmorillonite for stability enhancement of L-ascorbic acid
}

\author{
Yuan-Haun Lee ${ }^{\mathrm{a}, \mathrm{b}}$, Bor-Yann Chen ${ }^{\mathrm{c}, *}$, Kun-Yu Lin ${ }^{\mathrm{a}}$, \\ King-Fu Lin ${ }^{a}$, Feng-Huei Lin ${ }^{b}$ \\ ${ }^{a}$ Department of Material Science and Engineering, National Taiwan University, Taipei 106, Taiwan \\ ${ }^{\mathrm{b}}$ Institute of Biomedical Engineering, National Taiwan University, Taipei 106, Taiwan \\ ${ }^{\mathrm{c}}$ Department of Chemical and Materials Engineering, National I-Lan University, I-Lan 260, Taiwan
}

Received 1 October 2007; accepted 20 December 2007

\begin{abstract}
This study tended to construct new L-ascorbic acid (LAA) composites in low toxicity and high stability for feasible application. LAA is chemically very unstable, since it is easily oxidized into biologically inactive compounds naturally. Our finding showed that introduction of montmorillonite (MMT) could significantly attenuate its toxicity and to sustain the stability of LAA with economic feasibility for practical uses. In addition, as phosphoric acid was biologically compatible, it was used for the pretreatment of MMT to obtain a promising stabilization of LAA. Toxicity assessment also showed that MMT treated with low-concentration acids should be considered as biologically safe according to our assessment. Thus, using acid treated MMT to stabilize LAA in a long-term might be technically feasible for further uses.
\end{abstract}

(C) 2008 Taiwan Institute of Chemical Engineers. Published by Elsevier B.V. All rights reserved.

Keywords: L-Ascorbic acid (LAA); Montmorillonite; Dose-mortality analysis; Saccharomyces cerevisiae

\section{Introduction}

L-Ascorbic acid (LAA or vitamin C) has been shown to significantly stimulate the synthesis of collagen, especially in the skin, bone and blood vessels (Darr et al., 1993). As the skin naturally requires antioxidants to protect itself from detrimental effects of sunlight, LAA was predominantly used as the prime selection among biologically compatible antioxidants for skin care. LAA also played a crucial role to react with free radicals present in the environment and to inhibit the formation of melanin to against skin cancer and photoaging (Chang, 1998; Jagetia et al., 2007). Vitamin C and E were functioning synergistically; when vitamin $\mathrm{E}$ becomes oxidized by free radicals, it can be regenerated in the cellular membrane by vitamin C (LAA). In addition, LAA showed some promising antioxidant protection capability when skin was irradiated with solar-simulated UV irradiation (Lin et al., 2003). However, LAA is chemically very unstable, as it is easily oxidized into

\footnotetext{
* Corresponding author. Tel.: +886 $39357400 x 711$; fax: +88639357025. E-mail address: bychen@niu.edu.tw (B.-Y. Chen).
}

biologically inactive compounds (e.g., oxalic acid, L-xylonic acid, L-threonic acid, and L-lyxonic acid) under the exposure to air, light, moisture, heat, metallic ions, oxygen and bases (Kutsky, 1981). Therefore, the augmentation of biologically compatible and safe material(s) to stably maintain the activity of LAA for long-term use is apparently needed (Kwakye, 2000). As suggested in prior studies (Lee et al., 2005; Lin et al., 2002; Chen et al., 2006a), montmorillonite (MMT) was one of feasible candidates for such a purpose. The structure of MMT is an octahedral laminated sheet, sandwiched between tetrahedral silicate layers (Grim, 1968). Due to the excellent cation exchange property, MMT could form many nanocomposites with different organic compounds within its interlayer spacing of ca. 0.9$1.2 \mathrm{~nm}$ (Lin et al., 2002). Lee et al. (2005) also carried out the yeast and Wistar rats model to reveal that MMT is biologically safe and non-toxic as a drug carrier for further applications. For example, Lin et al. (2002) indicated that 5-fluorouracil (5-FU) could be successfully intercalated into the interlayer spacing of MMT as a feasible composite for the treatment of colorectal cancer. Although LAA/MMT composites might be biologically viable for possible further applications, the activity of LAA in composites still could not linger in a sufficient period of time. 


\section{Nomenclature}

$a_{i} \quad$ condensation coefficient

$A \quad$ the intercept of dose-mortality relation

$B$ the Hill slope (steepness or slope factor) of dosemortality relation

$d \quad$ the interplanar spacing of the clay layers $(\AA)$

DHLAA dehydro-L-ascorbic acid

2,3-DKLGA 2,3-diketo-L-gulonic acid

$E_{1} \quad$ the energy activation of desorption (J)

$\mathrm{EC}_{x} \quad$ effective concentration to provoke $x \%$ response $(\mathrm{mg} / \mathrm{L})$

$k \quad$ the slope of the straight line of $\ln \left(\mathrm{ABS}_{\mathrm{ABS}}\right)$ versus time $(1 / \mathrm{h})$

$K$ the rate of adsorbed molecules in per gain sample ( $\mathrm{mol} / \mathrm{g} \mathrm{Pa} \mathrm{s}$ )

LAA L-ascorbic acid (vitamin C)

MDLAA mono-dehydro-L-ascorbic acid

MMT montmorillonite

$n_{\mathrm{a}} \quad$ the total number of molecules adsorbed (mol/g)

$n_{\mathrm{m}}$ the number of molecules which would be adsorbed in a completed monolayer $(\mathrm{mol} / \mathrm{g})$

$\mathbf{P}$ the response (\%) corresponding to administered sample $\left(\mathbf{P}=1-\mathrm{VCC}_{\mathrm{VCC}}\right)$

$P \quad$ pressure $(\mathrm{Pa})$

$Q \quad$ irradiance $\left(\mathrm{W} / \mathrm{m}^{2}\right)$ striking the detector with a sample cell

$R \quad$ ideal gas constant $(\mathrm{J} / \mathrm{mol} \mathrm{K})$

$T \quad$ temperature $(\mathrm{K})$

TT toxicity threshold

VCC viable cell count (cells/mL)

$Y \quad$ probit unit

$Z$ the concentration of sample to be tested $(\mathrm{mg} / \mathrm{L})$

Greek symbols

$\lambda \quad$ wavelength (nm)

$\theta \quad$ glancing angle of incidence

$\theta_{i} \quad$ the fraction of the surface adsorbed gas molecules in the $i$ th layer

$v_{i} \quad$ the frequency of oscillation of the adsorbed molecules in the $i$ th layer $(1 / \mathrm{s})$

$v_{1} \times \mathrm{e}^{-E_{i} / R T}$ the number of molecules evaporating from a given site per second $(1 / \mathrm{s})$

Serpen and Gökmen (2007) indicated that the presence of reducing or oxidizing agents in the medium affected the reaction rate constant for reversible degradation kinetics of LAA. In addition, the scheme for LAA degradation (Davies et al., 1991) could be revealed as follows: (1) the two-step reversible conversion of LAA $\rightleftarrows$ mono-dehydro-L-ascorbic acid (MDLAA) $\rightleftarrows$ dehydro-L-ascorbic acid (DHLAA) (as shown below), where structures (a), (b), (c) and (d) denoted LAA, MDLAA, DHLAA, and 2,3-diketo-L-gulonic acid (2,3-DKLGA) respectively. (2) The final irreversible conversion DHLAA $\rightarrow$ 2,3-DKLGA caused the significant decay of LAA activity.

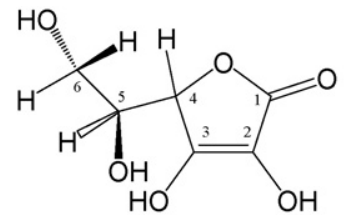

(a) L-ascorbic acid (LAA)

(d) 2,3-diketo-L-gulonic acid (2,3-DKLGA)

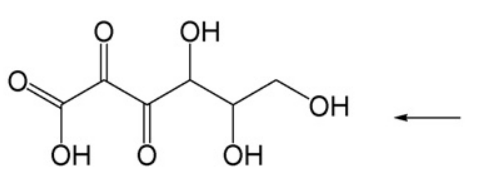

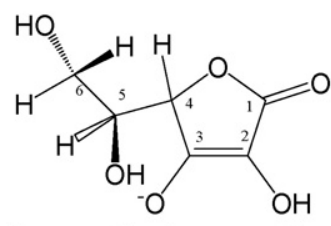

(b) mono-dehydro-L-ascorbic acid (MDLAA)
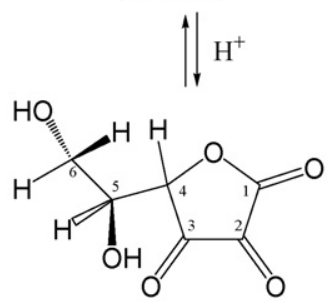
(DHLAA) (c) dehydro-L-ascorbic acid

Obviously, certain modification on LAA-containing composites should be carried out to stably maintain the activity of LAA for practical uses. That is, some engineered modification upon MMT/LAA composites is of course inevitable to maintain the activity of LAA in a longer period of time for safe applications. Therefore, the objectives of this feasibility study were not only to significantly attenuate the biotoxicity of augmented antioxidant, but also to considerably stabilize the activity of LAA with the aid of the phosphoric acid-treated LAA/MMT composites.

\section{Materials and methods}

\subsection{Materials and microorganisms}

MMT was purified by crude clay-bentonite obtained from Tai-Dong, Taiwan. The crude clay of bentonite in Tai-dong, Taiwan contained several kinds of clay (e.g., chlorite, kaolinite, illite, and MMT). MMT is about $30-40 \%$ by weight in unearthed bentonite. The purification process is briefly described as follows: $1 \mathrm{~kg}$ crude clay was dispersed in $4.0 \mathrm{~L}$ of distilled water and then maintained at room temperature for 3 days to be swelled up by all clay minerals. To obtain a better distribution of MMT to be resulted afterwards, the clay solution was then poured into a filtration tank and gently stirred at a rate of ca. $60 \mathrm{rpm}$ for 1 day. Particles left on the filtration tank were discarded and all the supernatant was then collected. The filtered solution was then centrifuged $(700 \times g)$ twice. The supernatant was discarded and precipitates were freeze-dried. The dried powder (size about $2.5 \mu \mathrm{m}$ ) was classified as pure MMT for further experimental use. L-Ascorbic acid (LAA; >99\% purity; Chung Chuan Industrial Chemicals Co., Ltd.) was used as the probing indicator of various composites and mixtures. Saccharomyces cerevisiae CCRC 22049 (purchased from Food Industry Research and Development Institute, Hsinchu) was used as the reporter microorganism for biotoxicity assessment. 


\subsection{Preparation of acid-treated MMT}

The deionized water was supplemented with MMT which was treated with various concentrations of phosphoric acid $\left(\mathrm{H}_{3} \mathrm{PO}_{4}\right.$; ca. 0.01-0.5 N). After $12 \mathrm{~h}$ well-mixing of MMT with $\mathrm{H}_{3} \mathrm{PO}_{4}$ at room temperature, deionized water was used to remove residual acid present in MMT mixtures and this procedure was carried out three times. Then, this MMT slurry was heated at $70{ }^{\circ} \mathrm{C}$ via dewatered drying for $12 \mathrm{~h}$ to obtain acid-treated MMT powders for study.

\subsection{XRD analysis}

The XRD patterns for the mineralogy of various MMT composite samples (in powders) were obtained by a RIGAKU Geigerflex diffractometer equipped with $\mathrm{Cu} K \alpha \quad \mathrm{X}$-ray radiation, operating at $40 \mathrm{kV}$ and $30 \mathrm{~mA}$. The diffraction patterns were ranged in angles $(2 \theta)$ of $4-45^{\circ}$ running at a scanning rate and counting step size of $1.2^{\circ} / \mathrm{min}$ and $0.02^{\circ}$, respectively. As the $\left(\begin{array}{lll}0 & 0 & 1\end{array}\right)$ diffraction peak of MMT appears at $2 \theta=7.2^{\circ}$ in this angle range, the interlayer spacing at the $\left(\begin{array}{lll}0 & 0 & 1\end{array}\right)$ plane can thus be determined from the XRD response using Bragg's law.

\subsection{BET analysis and calculation}

The BET (named after Brunauer, Emmett, Teller) analysis for surface areas were carried out via nitrogen adsorption at $77 \mathrm{~K}$ using a NOVA2000, Quantachrome Co. surface area analyzer. All samples were outgassed under vacuum at $120^{\circ} \mathrm{C}$ for twenty minutes before $\mathrm{N}_{2}$ adsorption. Surface areas were determined via the BET theory in the linear form (Gregg and Sing, 1967): $\left(P / P_{0}\right) /\left(n_{\mathrm{a}}\left(1-P / P_{0}\right)\right)=\left(1 / n_{\mathrm{m}} c\right)+\left(c-1 / n_{\mathrm{m}} C\right)$ $\left(P / P_{0}\right)$ (refer to Appendix A for details), where $P / P_{0}$ : relative pressure; $n_{\mathrm{m}}$ was determined from the slope, $c$ : constant related to the net heat of monolayer adsorption of a linear relationship $P / P_{0}$ versus $\left(P / P_{0}\right) /\left(W\left[1-\left(P / P_{0}\right)\right]\right) ; n_{\mathrm{a}}$ : the amount of gas adsorbed. Here, as the technique of "BET multiple-point measurement" was used for determining surface areas of heated samples of tested materials (e.g., MMT). In addition, only six data points within the range of $P / P_{0}$ at $0.05-0.3$ were selected for such calculations of surface areas (Helmy et al., 1999).

\subsection{Biotoxicity assessment}

Dose response analysis of tested material (e.g., MMT, LAA) using $S$. cerevisiae as probing bacterium was conducted as described elsewhere (Lee et al., 2005). Biotoxicity assessment was specially designated through a modification of dosemortality analysis (Chen, 2006) as follows: the tested sample (e.g., LAA, MMT and acid treated MMT) was first sterilized via moist-heat method $\left(121^{\circ} \mathrm{C}\right.$ at 15 psi for $\left.20 \mathrm{~min}\right)$ to exclude the presence of unwanted microbial contaminants (or $0.2 \mu \mathrm{m}$ sterile filtration if heat sensitive) originally present in tested material. The "apparent" concentration of sample defined herein was the concentration of tested samples and their serial diluents well mixed with sterile deionized water. Note that phosphate buffered saline (PBS) solution, which is regularly used for biological assay, was not applicable for serial dilution of our sample solutions, since metal phosphate precipitates might be formed in the presence of metal ions to interfere with results.

The initial concentration $C_{0}$ for toxicity tests of samples was appropriately chosen (e.g., $20.0 \mathrm{~g} / \mathrm{L}$ ). Serial-half dilution of initial concentration $C_{0}$ (i.e., $(1 / 2) C_{0},(1 / 4) C_{0},(1 / 8) C_{0}$, $\left.(1 / 16) C_{0},(1 / 32) C_{0}, \ldots,\left(1 / 2^{n}\right) C_{0}\right)$ was carried out by using $50 \mathrm{~mL}$ sample solution or its derived diluents mixed with $50 \mathrm{~mL}$ deionized water. The 9-mL resulted serial diluents (RSD) were all placed in sterile test tubes for quantification of viable cells afterwards. One milliliter of freshly harvested TS was then well shook with RSD ca. 20 times through a 35-cm arc elbow motion to form serial plate-count diluents (SPCD). Meanwhile, $1 \mathrm{~mL}$ fresh TS mixed with $9 \mathrm{~mL}$ deionized water was chosen as the sample-free control. The numbers of viable $S$. cerevisiae in SPCD or the control were estimated by the standard plate count method. Standard plate count in YM medium was carried out as follows: SPCD were serially diluted with deionized water immediately after sampling, and then appropriate volumes (ca. $0.1-0.2 \mathrm{~mL}$ ) of SPCD were well spread onto agar Petri plates. Note that all cells in SPCD would be assumed viable and culturable on YM-medium plates due to fresh preparation of cells in maximal viability for all steps. The YM-medium plates were then incubated at $30{ }^{\circ} \mathrm{C}$ for ca. $16-24 \mathrm{~h}$ to form observable colonies for enumeration. Plates with between 30 and 300 colonies are considered statistically appropriate for counting. The microbial population in the original RSD was calculated using the following formula (VCC: viable cell count):

\section{Cells per liter of broth (VCC) \\ $=\frac{\text { number of colonies }}{\text { amount plated } \times \text { dilution factor }}$}

To obtain quantitative toxicity for comparison, $\mathrm{VCC}_{0}$ was chosen as the VCC at sample-free deionized water control. The ratio $\mathrm{VCC} / \mathrm{VCC}_{0}$ of 0 and 1 directly indicated complete inhibition and no inhibitory toxicity to $S$. cerevisiae cell, respectively. The unity of this ratio simply suggests that the present toxicity of this diluent at this concentration nearly equals the toxicity of deionized water (i.e., "zero" toxicity). The concentration range for the ratio jumped from 1.0 to 0 in dose-mortality curves is defined as the "toxicity threshold" (TT) range. The TT ranges of various samples in dose-mortality curves can provide obvious figures of their toxicity ranking. For example, if the TT range for sample A is much less than that for sample B, sample A is inevitable much more toxic than sample $\mathrm{B}$, indicating that much higher dilution factor must be carried out for sludge A to reach "zero" toxicity as same as control (i.e., deionized water).

\subsection{Dose-mortality analysis}

Probit analysis (Chen, 2006) was adopted to reveal dose mortality curves of various tested samples. The probit model 
postulates that the tolerance capacity of indicator microorganisms $S$. cerevisiae in response to the suspected toxic material in a given population is log-normal distribution. The mid-point effective concentration (i.e., $\mathrm{EC}_{50}$ ) of the tested material on its dose-mortality curve was usually selected as a comparative basis for toxicity comparison. This is simply because it is usually easier to interpolate the mid-point $\mathrm{EC}_{50}$ accurately than to make extrapolated estimates of critical $\mathrm{EC}_{x}$ (e.g., $\mathrm{EC}_{0}$, $\mathrm{EC}_{100}$ ). Semilogarithmic plot of toxic-species concentration versus the provoked response is assumed to reveal a linear relation. Probit model converts sigmoid-shaped dose-mortality curve into a linear normal equivalent deviation (NED) scale. For example, the $50 \%$ and $84.1 \%$ response correspond to the NED scale in 0 and 1 , respectively. In addition, probit unit in the model equals NED scale plus 5 . The conversion formulae are shown as follows:

$Y=A+B \log Z$,

$\mathbf{P}=\frac{1}{2}\left\{1+\operatorname{erf}\left(\frac{Y-5}{\sqrt{2}}\right)\right\}$,

$\operatorname{erf}(x) \equiv \frac{2}{\sqrt{\pi}} \int_{0}^{x} \mathrm{e}^{-\xi^{2}} \mathrm{~d} \xi$

where $A$ and $B$ denote the intercept and the Hill slope (i.e., steepness or slope factor) of dose-mortality relation, $Z$ and $Y$ are composite sample concentration (ppm or $\mathrm{mg} / \mathrm{L}$ ) and probit unit, respectively; $\mathbf{P}$ is the response (\%) corresponding to administered sample, $\operatorname{erf}(x)$ is an error function. Note that the response variable is normalized to be located between 0 and 1 . The conversion relation between the probit unit and provoked response is listed elsewhere (refer to Table 1 in Chen et al., 2006 b). For example, $55 \%$ and $85 \%$ of toxic response correspond to probit unit of 5.13 and 6.04, respectively. The toxicity responses are determined by $\mathbf{P}=1-\left(\mathrm{VCC} / \mathrm{VCC}_{0}\right)$, where $\mathrm{VCC}$ and $\mathrm{VCC}_{0}$ denoted the viable cell count remained in the sample culture and deionized water, respectively. The slope $B$ of the dose-mortality curve also provides information which is vital in evaluating potential biotoxicity of tested materials to indicator microorganism $S$. cerevisiae. The Hill slope $B$ may be steep (e.g., $B>1$ ) or shallow (e.g., $B<1$ ), indicating that the effective concentration range (i.e., TT or the range from $\mathrm{EC}_{0}$ to $\mathrm{EC}_{100}$ ) is narrow (i.e., steep slope) or wide (shallow slope), respectively. The steep slope means that, within the $S$. cerevisiae, there is only a small difference between the concentration that is lethal for the most susceptible cells and the concentration that is lethal for the most resistant cells. If the concentration of

Table 1

The $d_{0} 0_{1}$ space of MMT and varying acid concentration treated montmorillonite

\begin{tabular}{lcc}
\hline & $2 \theta\left({ }^{\circ}\right)$ & $d_{001}(\AA)$ \\
\hline MMT & 7.20 & 12.26 \\
$0.01 \mathrm{~N}$ treated MMT & 5.70 & 15.49 \\
$0.05 \mathrm{~N}$ treated MMT & 5.72 & 15.43 \\
$0.1 \mathrm{~N}$ treated MMT & 5.70 & 15.49 \\
$0.5 \mathrm{~N}$ treated MMT & 5.70 & 15.49 \\
\hline
\end{tabular}

toxic materials exceeded $\mathrm{EC}_{0}$ (i.e., threshold), only a slightly increase in the concentration might result in a remarkable rise in mortality of $S$. cerevisiae cells and even more severe in compete loss of cellular viability (i.e., $\mathrm{EC}_{100}$ ).

\subsection{Analysis of LAA activities in MMT and acid-treated MMT mixtures}

Single-beam scanning UV/VIS spectrophotometer (JASCO $\mathrm{V}-550$ ) was obtained time-course spectra a maximal absorbance at $\lambda_{\max }=265 \mathrm{~nm}$ to determine spectra and measure absorbance for LAA. The absorbance (ABS) at $265 \mathrm{~nm}$, which is proportional to LAA concentration, was obtained by $\mathrm{ABS}=$ $\log \left(Q_{0} / Q\right)=-\log T$, where $Q_{0}$ and $Q$ denoted the irradiance $\left(\mathrm{W} / \mathrm{m}^{2}\right)$ striking the detector with a reference cell and sample cell in the sample compartment, respectively. As free LAA in aqueous solution can be easily oxidized to lose its activity, the experimental design for stability assessment of LAA composites with various concentrations of supplemented materials (e.g., MMT or acid treated MMT) was carried out as follows: First, as a lower concentration of LAA was significantly stabilized to mask the decay profile of LAA, LAA was intentionally selected at the concentration of $0.001 \%(\mathrm{w} / \mathrm{v})(\mathrm{ca}$. $\mathrm{ABS}_{265 \mathrm{~nm}}=0.8$ ) to increase the sensitivity for this evaluation.

The stabilizing capability of supplemented MMT or acid treated MMT at various concentrations could be determined in term of the rate constant $k$. Then, at room temperature, the absorbance at the wavelength of $265 \mathrm{~nm}$ was determined at various times (e.g., 0, 3, 6, 9, $12 \mathrm{~h}$ ) for all designated cases. The initial decay rate of the activity of LAA was assumed to follow a first-order reaction (i.e., $\left.(\mathrm{dABS} / \mathrm{d} t)=-k \cdot \mathrm{ABS} ; \mathrm{ABS}_{(0)}=\mathrm{ABS}_{0}\right)$; thus, a plot of $\ln \left(\mathrm{ABS} / \mathrm{ABS}_{0}\right)$ versus time gave a straight line with a slope of $-k$. Note that the larger value of slope $k$ simply suggested a less stability for the tested sample.

\section{Results and discussion}

Montmorillonite is a kind of bioinert aluminosilicate (or clay) mineral with fine grain and large inter-planar spacing, in particular located in the $\left(\begin{array}{lll}0 & 0 & 1\end{array}\right)$ plane, showing a superior capability to intercalate large molecules into the interlayer space at the $\left(\begin{array}{lll}0 & 0 & 1\end{array}\right)$ plane. It was shown that the $\left[\begin{array}{lll}0 & 0 & 1\end{array}\right]$ diffraction peak of the montmorillonite appears at $2 \theta=7.2^{\circ}$ (Table 1). The characteristic basal spacing $d$ is $12.4 \AA$ as calculated from Bragg's Law (Eq. (4)) as follows:

$n \lambda=2 d \sin \theta$,

where $n, \lambda, \theta$, and $d$ are an integer, the wavelength, the glancing angle of incidence and the interplanar spacing of the clay layers, respectively. For example, plugging $n=1, \lambda=1.54$ $\mathrm{nm}, \theta=7.2^{\circ}$ for copper target of X-ray into Eq. (4), we might obtain $d=12.26 \AA$ for interplanar spacing at the $\left(\begin{array}{lll}0 & 0 & 1\end{array}\right)$ plane. As indicated in Table 1 and Fig. 1, changes in the glancing angle of incidence in X-ray diffraction patterns revealed that interplanar spacing increased from 12.26 to $15.49 \AA$ when MMT was treated by $\mathrm{H}_{3} \mathrm{PO}_{4(\mathrm{aq})}$. The changes in the glancing angle were likely due to the intercalation of protons and phosphate 


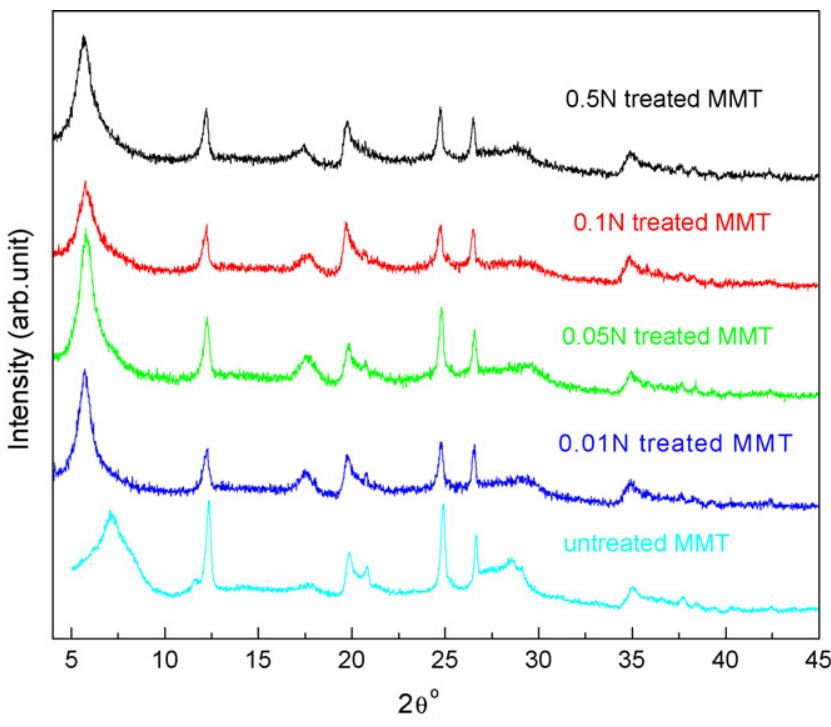

Fig. 1. X-ray diffraction patterns of montmorillonite and acid treated montmorillonite. Significant increases in interplanar spacing after acid-treatment were revealed.

ions into interlayer space. However, no significant increases in interplanar spacing were found at larger concentrations of $\mathrm{H}_{3} \mathrm{PO}_{4}$ used for acid treated MMT, indicating that a maximal spacing was apparently achieved at $0.01 \mathrm{~N}$ acid-treated MMT.

As indicated in the decay profile of LAA (Fig. 2), the augmentation of MMT was viable to stabilize LAA compared to LAA alone. Therefore, when the content of MMT gradually increased (except at $1.0 \%$ ), the $k$ value decreased significantly likely due to protection of MMT from the oxidation of LAA in air exposure. However, this exception of $1 \%(\mathrm{w} / \mathrm{v})$ was likely due to large cation exchange capacity of MMT leading to a negative interference (or confounding interactions) to this protection. In addition, when MMT was added, the proton would be neutralized by hydroxide ion from MMT, resulting in increases in the $\mathrm{pH}$ levels. For example, $\mathrm{pH}$ values for LAA alone, MMT $(0.1 \%)$,

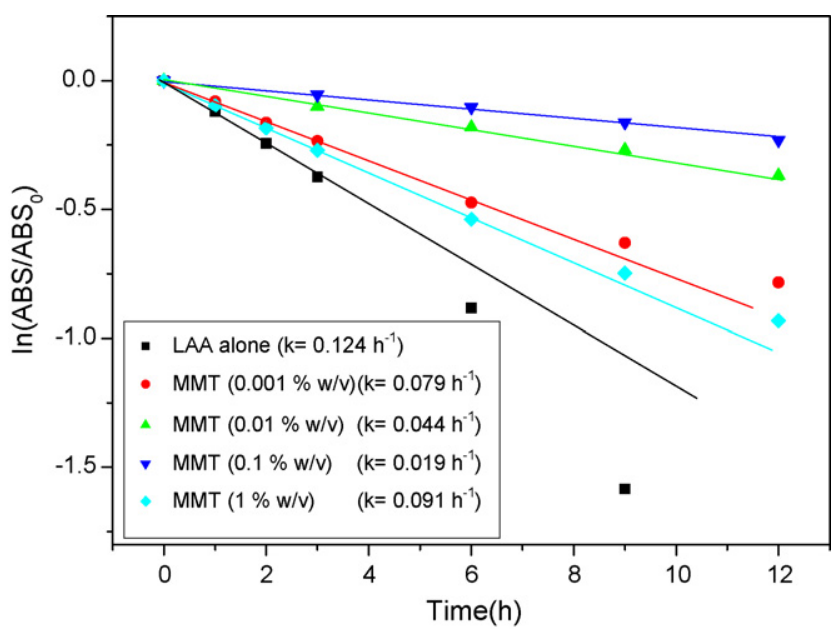

Fig. 2. Decay profiles of activities of LAA in various MMT concentrations (initial LAA at $0.001 \%, \mathrm{w} / \mathrm{v}$ ). The first-order kinetics for decay profiles of LAA activities was assumed to be satisfied for initial rate determination.

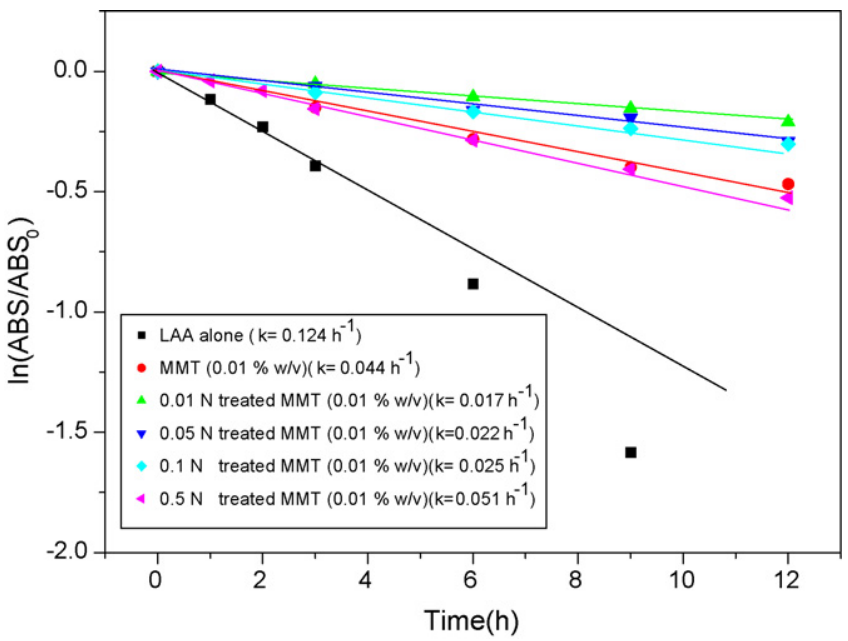

Fig. 3. Decay profile characteristics of LAA $0.001 \%(\mathrm{w} / \mathrm{v})$ in various acid concentrations treated MMT. Decay profiles of activities of LAA in acid-treated MMT by different concentration of $\mathrm{H}_{3} \mathrm{PO}_{4}$. The initial LAA and MMT were at $0.001 \%(\mathrm{w} / \mathrm{v})$, and $0.01 \%(\mathrm{w} / \mathrm{v})$, respectively. Assuming the first-order kinetics for decay profiles of LAA activities was satisfied for initial rate determinations.

MMT (1\%) were 3.65, 3.75, 4.27, respectively (data not shown). Note that the formation of intermediates and products in significant amounts to violate first-order kinetics might be the main reason of large differences with predications of the decay profile.

Evidently, the stability of LAA strongly associated with a large adsorption capacity and a high surface area of MMT $\left(758 \mathrm{~m}^{2} / \mathrm{g}\right.$; Helmy et al., 1999), as MMT could mask with certain portion of dissolved oxygen to impede the oxidation of LAA during air exposure. In addition, when LAA mixed with $0.01 \mathrm{~N}$ phosphoric acid treated MMT $(0.01 \% \quad(\mathrm{w} / \mathrm{v})$, $\left.k=0.017 \mathrm{~h}^{-1}\right)$, the decay rate of LAA was smaller than that of LAA mixed with intact MMT $\left(0.01 \%(\mathrm{w} / \mathrm{v}), k=0.044 \mathrm{~h}^{-1}\right.$; ca., 2.6-fold) (Fig. 3). Moreover, the decay constant of LAA mixed with MMT $(0.01 \%$, w/v) treated with $0.01 \mathrm{~N}$ phosphoric acid was lower (ca. 12\%) than higher amount intact MMT

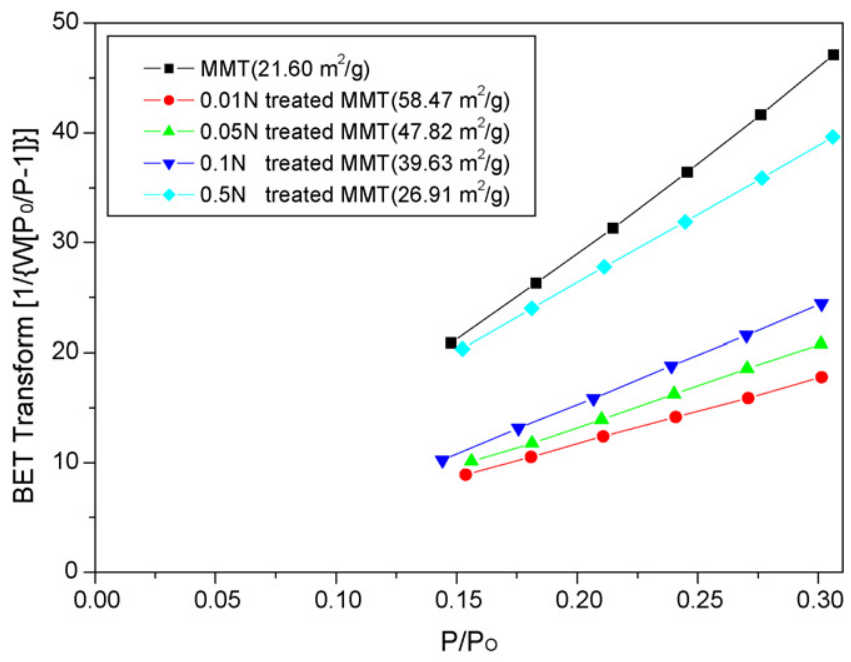

Fig. 4. The experimental relationship of $\left(P / P_{0}\right) / w\left(1-P / P_{0}\right)$ versus $P / P_{0}$ for MMT and different acid-treated MMT using BET analysis. 


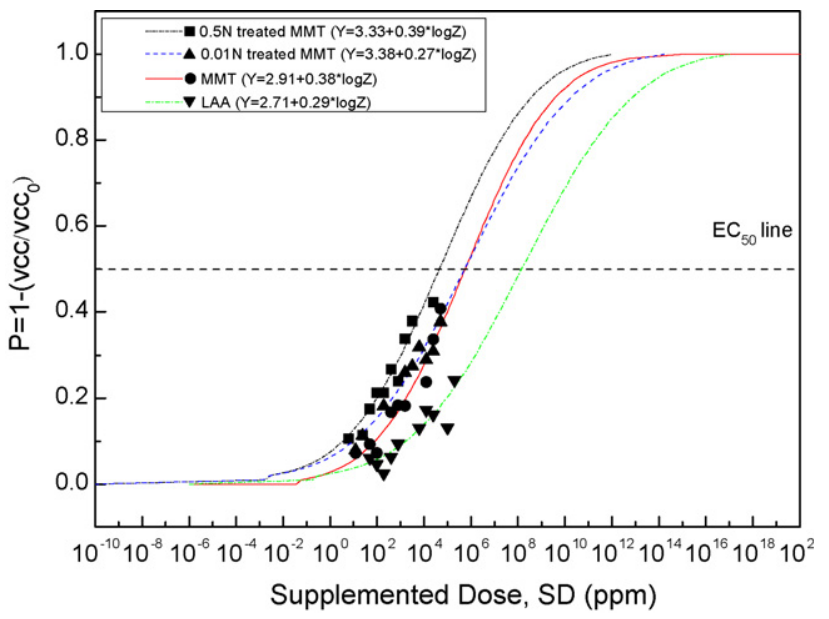

Fig. 5. Dose-mortality curves for $S$. cerevisiae using different materials as maxtrices. In these curves, all of the $B$ values less than unity and predicted $\mathrm{EC}_{50}$ greater than $10^{4}$ ppm suggested insignificant toxicities of tested materials.

$\left(0.1 \%(\mathrm{w} / \mathrm{v}), k=0.019 \mathrm{~h}^{-1} ;\right.$ Fig. 3). Fig. 4 indicated that MMT treated with low concentration acid provided a higher surface area than intact/untreated MMT (e.g., $58.47 \mathrm{~m}^{2} / \mathrm{g}(0.01 \mathrm{~N}$ treated MMT) $>21.60 \mathrm{~m}^{2} / \mathrm{g}$ (intact MMT)). When the acid concentration for the treatment increased, the surface area of MMT gradually decreased. This decrease was perhaps due to a higher concentration of $\mathrm{Al}^{3+}$ in MMT could be replaced by a higher concentration of $\mathrm{H}^{+}$ions. In addition, released $\mathrm{Al}^{3+}$ could aggregate MMT particles together (Hendershot and Lavkulich, 1983), leading to some degree of decrease in surface area of MMT. Moreover, the coating of smectites with trivalent ions (e.g., $\mathrm{Al}^{3+}$ and $\mathrm{Fe}^{3+}$ ) were very effective in coagulating smectite particles. However, Al(III)-coated clays had lower surface areas than the Fe(III)-coated samples. Therefore, release and adsorption of $\mathrm{Al}^{3+}$ ions might be an alternative explanation for the low surface area of montmorillonite at lower $\mathrm{pH}$ values (Table 2). The increased $\mathrm{Fe}^{3+}$ ions might mask some of the protective power of MMT to LAA, resulting in certain acceleration of LAA oxidation (Serpen and Gökmen, 2007). These all supported that there existed an optimal acid concentration for a maximal stabilization (e.g., $0.01 \mathrm{~N}$ acid treated MMT $(0.01 \%, w / v)$; Fig. 3). To get a grasp of biosafety profile of MMT-LAA composition for possible industrial applications, dose-mortality curves of S. cerevisiae (Fig. 5) were carried out, showing that MMT treated with a lower acid treated concentration $(0.01 \mathrm{~N})$ had a less toxic impact upon the growth of $S$. cerevisiae (i.e., $\mathrm{EC}_{50}(\mathrm{ppm})$ : $\operatorname{MMT}(0.5 \mathrm{~N})=$

Table 2

The EDS element analysis of MMT and acid treated MMT (\%) (values indicated averages of six replicates in chemical analysis)

\begin{tabular}{llllllllll}
\hline & $\mathrm{O}$ & $\mathrm{Na}$ & $\mathrm{Mg}$ & $\mathrm{Al}$ & $\mathrm{Si}$ & $\mathrm{P}$ & $\mathrm{K}$ & $\mathrm{Ca}$ \\
\hline $0 \mathrm{~N}$ & 35.01 & 0.89 & 1.52 & 21.55 & 34.88 & 0.13 & 2.90 & 0.85 & 2.46 \\
$0.01 \mathrm{~N}$ & 38.08 & 0.84 & 1.60 & 20.72 & 33.17 & 0.19 & 2.64 & 0.67 \\
$0.05 \mathrm{~N}$ & 37.98 & 0.40 & 1.58 & 21.60 & 33.17 & 0.28 & 2.64 & 0.56 \\
$0.1 \mathrm{~N}$ & 39.07 & 0.38 & 1.63 & 20.86 & 33.20 & 0.60 & 2.20 & 0.65 \\
$0.5 \mathrm{~N}$ & 43.63 & 0.34 & 1.47 & 19.68 & 30.61 & 0.85 & 2.05 & 0.47 \\
\hline
\end{tabular}

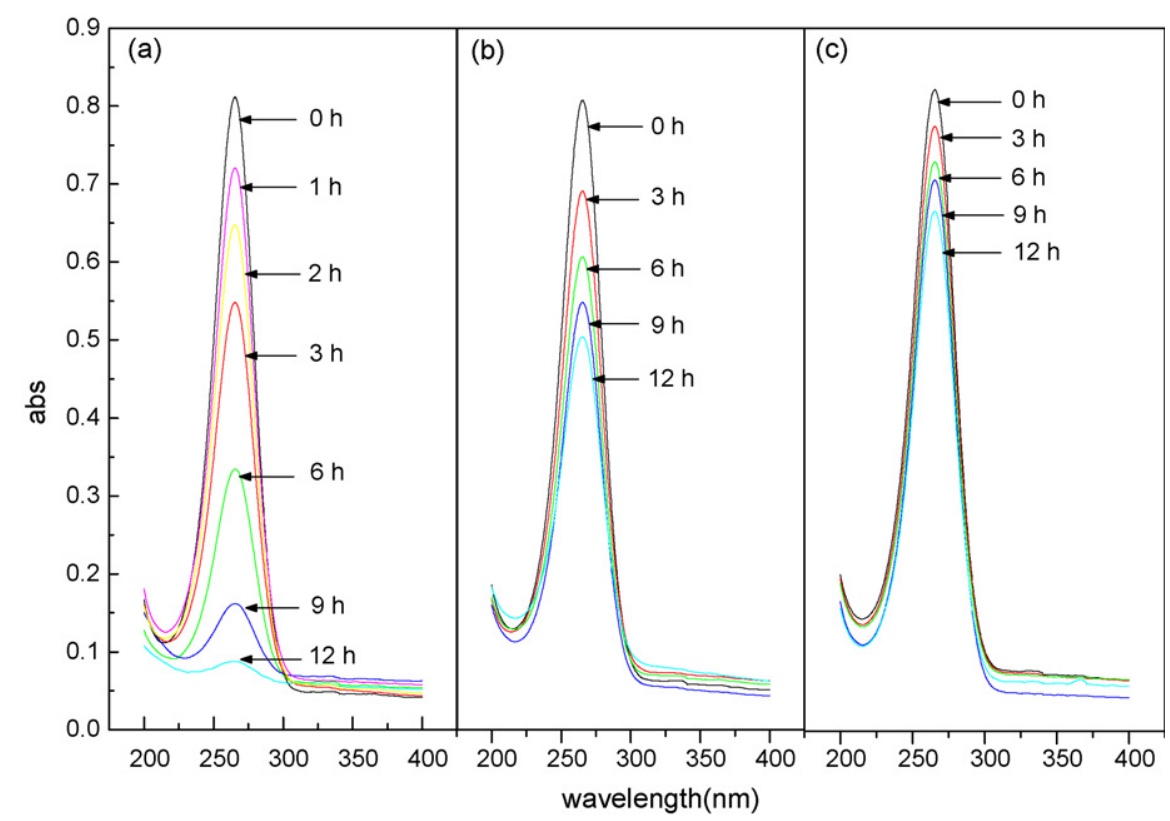

Fig. 6. The decay UV spectra of initial L-ascorbic acid at $0.001 \%$ (w/v) (a) without supplemented MMT, (b) with supplemented MMT (0.01\%, w/v), and (c) with supplemented acid treated MMT $(0.01 \%$, w/v). The augmentation of MMT impeded significant losses of LAA activities. 
$\left.5.46 \times 10^{4}, \operatorname{MMT}(0.01 \mathrm{~N})=6.77 \times 10^{5}, \mathrm{MMT}=5.67 \times 10^{5}\right)$. Note that the extremely high levels of predicted $\mathrm{EC}_{50}$ (ca. $>10^{4} \mathrm{ppm}$ ) only showed the toxicity ranking for MMT for a quantitative comparison, but very possibly exceeded the saturated solubility of MMT. Thus, MMT treated with a relatively lower concentration acid should be considered to be a biologically safe material for further uses.

\section{Conclusion}

Evidently, the supplementation of MMT significantly lowered the decay rate of LAA about 6.5 -fold (i.e., $k$ value (LAA $(0.001 \%, \mathrm{w} / \mathrm{v}))=0.124 \mathrm{~h}^{-1} ; k(\operatorname{LAA}(0.001 \%, \mathrm{w} / \mathrm{v})+\mathrm{MMT}$ $(0.1 \%, w / v))=0.019 \mathrm{~h}^{-1} ;$ Fig. 2$)$. The LAA with MMT (treated by a relatively low concentration phosphoric acid) was performed more stably than an intact MMT (0.017 vs. $0.044 \mathrm{~h}^{-1} ; 2.6$-fold decrease in Fig. 3). In $12 \mathrm{~h}$ (i.e., a typical period for practical application of LAA), the LAA $(0.001 \%$, w/v) almost completely lose its activity (ca. 97\% loss compared to LAA alone; Fig. 6). In contract, LAA mixed with $0.01 \mathrm{~N}$ treated MMT $(0.01 \%$, w/v) decreased only $26 \%$ in activity $(0.01 \mathrm{~N}$ treated MMT; Fig. 6). As stated herein, the decrease in decay rate constant for LAA activity was likely due to the shielding effect of MMT to prevent LAA significantly exposed to air. Toxicity assessment also showed that MMT treated with low-concentration acids should be considered as biologically safe according to our assessment. Thus, using MMT or acid-treated MMT to stabilize LAA in a long-term might be technically feasible for further uses.

\section{Acknowledgements}

Partial financial supports (NSC 96-2221-E-197-012, NSC 95-2221-E-197-005, NSC 94-2214-E-197-003) from National Science Council, Taiwan, ROC, for this study are gratefully acknowledged. The authors also thank anonymous reviewers for their significant comments.

\section{Appendix A. Theoretical background for BET analysis}

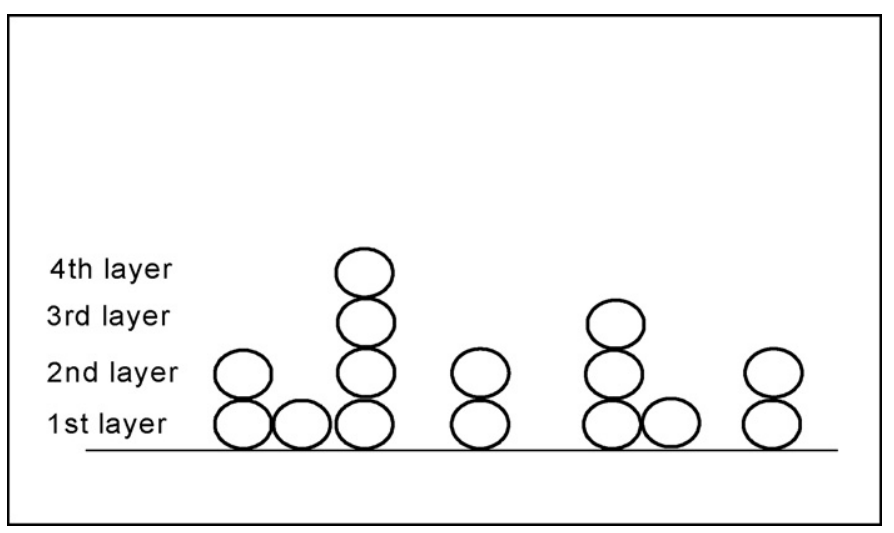

The diagram of gas adsorption using BET model in this study was chosen (Gregg and Sing, 1967).

\section{A.1. Estimation of the adsorption capacity in individual layers}

The rate of condensation of molecules into the first layer per second will be

$n_{1}=K \times P \times a_{1} \times \theta_{0}$

The rate of evaporation of molecules from the first layer per second will be

$n_{1}^{\prime}=n_{\mathrm{m}} \times \theta_{1} \times v_{1} \times \mathrm{e}^{-E_{1} / R T}$.

If an equilibrium is assumed to be achieved, we have $n_{1}=n_{1}^{\prime}$ as follows:

$K \times P \times a_{1} \times \theta_{0}=n_{\mathrm{m}} \times \theta_{1} \times v_{1} \times \mathrm{e}^{-E_{1} / R T}$.

Similarly, for molecules in second layer one may write

$K \times P \times a_{2} \times \theta_{1}=n_{\mathrm{m}} \times \theta_{2} \times v_{2} \times \mathrm{e}^{-E_{2} / R T}$

Similar equations might be applicable for the $i$ th layer in the following:

$K \times P \times a_{i} \times \theta_{i-1}=n_{\mathrm{m}} \times \theta_{i} \times v_{i} \times \mathrm{e}^{-E_{i} / R T}$

Brunauer, Emmett and Teller made assumptions as follow: (1) the heat of adsorption all layers above the first is equal to the latent heat of condensation (i.e., $E_{2}=E_{3}=\ldots E_{i}=L$ ), (2) the evaporation-condensation constants in all layers above the first are identical (i.e., there is no interactions of evaporation-andcondensation in all layers $)$, so that $\left(v_{2} / a_{2}\right)=\left(v_{3} / a_{3}\right)=\ldots\left(v_{i} / a_{i}\right)$. According to Eq. (A-3), we can obtain

$\frac{\theta_{1}}{\theta_{0}}=\frac{K \times a_{1} \times P}{n_{\mathrm{m}} \times v_{1}} \mathrm{e}^{L / R T}=\alpha$,

That is,

$\theta_{1}=\alpha \times \theta_{2}$

Similarly, Eq. (A-4) could be written as

$\frac{\theta_{2}}{\theta_{1}}=\frac{K \times a_{2} \times P}{n_{\mathrm{m}} \times v_{2}} \mathrm{e}^{L / R T}=\beta$

$\theta_{2}=\beta \times \theta_{1}=\alpha \times \beta \times \theta_{0}$

Similar formulations can be resulted in

$\theta_{3}=\beta \times \theta_{2}=\alpha \times \beta^{2} \times \theta_{0}$

.

$\theta_{i}=\alpha \times \beta^{i-1} \times \theta_{0}$

If $c$ is used to replace $\alpha / \beta$, we may have Eq. (A-10) as follows:

$\theta_{i}=c \times \beta^{i} \times \theta_{0}$

\section{A.2. Summation of capacity for gas adsorption}

Now as already stated the total adsorption for all layers is $n_{\mathrm{a}}=n_{\mathrm{m}}\left(\theta_{1}+2 \theta_{2}+3 \theta_{3}+\ldots+i \theta_{i}\right)_{i \rightarrow \infty}$ 
With the aid of the relationships implied in Eq. (A-10), this may be rewritten

$$
\begin{aligned}
n_{\mathrm{a}} & =n_{\mathrm{m}} \times c \times \theta_{0} \times\left(\beta+2 \beta^{2}+3 \beta^{3}+\ldots+i \beta^{i}\right)_{i \rightarrow \infty} \\
& =n_{\mathrm{m}} \times c \times \theta_{0} \times \frac{\beta}{(1-\beta)^{2}}
\end{aligned}
$$

\section{A.3. Model formulation}

According to the postulate, one may obtain

$\theta_{0}=1-\left(\theta_{1}+\theta_{2}+\theta_{3}+\ldots+\theta_{i}\right)_{i=\infty}=1-\sum_{i=1}^{\infty} \theta_{i}$

From Eq. (A-10), we obtain

$\theta_{0}=1-c \times \theta_{0} \times \sum_{i=1}^{i=\infty} \beta_{i}=1-c \times \theta_{0} \times\left(\frac{\beta}{1-\beta}\right)$

Substituting this value of $\theta_{0}$ into Eq. (A-11)

$$
\begin{aligned}
\frac{n_{\mathrm{a}}}{n_{\mathrm{m}}} & =\frac{c \times \beta}{(1-\beta)^{2}(1+c \times \beta /(1-\beta))} \\
& =\frac{c \times \beta}{(1-\beta) \times(1-\beta+c \times \beta)}
\end{aligned}
$$

As known, the adsorbate vapor condenses as a liquid when its saturated vapor pressure is reached, i.e., for $P=P_{0}, n_{\mathrm{a}} / n_{\mathrm{m}}=\infty$.

According to Eq. (A-13), the value of $\beta$ is then assumed to be unity, so that

$\beta=1$

Eq. (A-8) thus becomes

$\frac{K \times a_{2}}{n_{\mathrm{m}} \times v_{2}} \times \mathrm{e}^{L / R T} \times P_{0}=1$

Comparison of Eqs. (A-8) and (A-15) then gives

$\beta=\frac{P}{P_{0}}$

Eq. (A-11) thus becomes

$$
\begin{aligned}
& \frac{n_{\mathrm{a}}}{n_{\mathrm{m}}}=\frac{c \times\left(P / P_{0}\right)}{\left(1-\left(P / P_{0}\right)\right)\left(1+\left((c-1) /\left(P / P_{0}\right)\right)\right)} \\
& \text { or simplify as } \frac{P / P_{0}}{n_{\mathrm{a}} \times\left(1-\left(P / P_{0}\right)\right)}=\frac{1}{n_{\mathrm{m}} \times c}+\frac{c-1}{n_{\mathrm{m}} \times c} \times \frac{P}{P_{0}}
\end{aligned}
$$

Here, as $n_{\mathrm{a}}$ denoted the total number of molecules adsorbed (mol/g), one may simply convert $n_{\mathrm{a}}$ in terms of $W$ (total weight of gas adsorbed by unit weight of tested material; g/g) and $Z$ (the molecular weight of adsorbed gas; g/mol) as $n_{\mathrm{a}}=W / Z$.
Then, the formula of BET analysis could be obtained as follows:

$\frac{Z \times P / P_{0}}{W \times\left(1-\left(P / P_{0}\right)\right)}=\frac{1}{n_{\mathrm{m}} \times c}+\frac{c-1}{n_{\mathrm{m}} \times c} \times \frac{P}{P_{0}}$

\section{References}

Chang, L. C., Application of Cosmetics Inspecting, p. 243, Fayfar Publishing Co., Ltd., Taiwan (1998).

Chen, B. Y., Y. H. Lee, W.-C. Lin, F.-H. Lin, and K.-F. Lin, "Understanding the Characteristics of L-Ascorbic Acid/Montmorillonite Nanocomposite: Chemical Structure and Biotoxicity," Biomed. Eng. Appl. Basis Commun., 18, 30 (2006a)

Chen, B. Y., C. H. Wu, and J. S. Chang, "An Assessment of the Toxicity of Metals to Pseudomonas aeruginosa PU21 (Rip64)," Bioresour. Technol., 97, 1880 (2006b).

Chen, B. Y., "Toxicity Assessment of Aromatic Amines to Pseudomonas luteola: Chemostat Pulse Technique and Dose-Response Analysis," Process Biochem., 41 (7), 1529 (2006).

Darr, D., S. Combs, and S. Pinnell, "Ascorbic Acid and Collagen Synthesis: Rethinking a Role for Lipid Peroxidation," Arch. Biochem. Biophys., 307 (2), 331 (1993).

Davies, M. B., J. Austin, and D. A. Patridge, VITAMIN C: Its Chemistry and Biochemistry, p. 26, Royal Society of Chemistry, Paperbacks, Cambridge, U.K. (1991).

Gregg, S. J. and K. S. W. Sing, Adsorption Surface Area and Porosity, p. 36, Academic Press, New York, U.S.A. (1967).

Grim, R. E., Clay Mineralogy, 2nd Ed., p. 39, McGraw-Hill, New York, U.S.A. (1968).

Helmy, A. K., E. A. Ferreiro, and S. G. de Bussetti, "Surface Area Evaluation of Montmorillonite," J. Colloid Interface Sci., 210, 167 (1999).

Hendershot, H. W. and M. L. Lavkulich, "Effect of Sesquioxide Coating on Surface Charge of Standard Mineral and Soil Samples," Soil. Sci. Soc. Am. J., 47, 1252 (1983).

Jagetia, G. C., G. K. Rajanikant, and K. V. N. Mallikarjun, "Ascorbic Acid Increases Healing of Excision Wounds of Mice Whole Body Exposed to Different Doses of $\gamma$-Radiation," Burns, 33, 484 (2007).

Kutsky, R. J., Handbook of Vitamins, Minerals, and Hormones, 2nd Ed., p. 252, Van Nostrand Reinhold, New York, U.S.A. (1981).

Kwakye, J. K., "The Use of Stabilizers in the UV Assay of Ascorbic Acid," Talanta, 51, 197 (2000).

Lee, Y. H., T. F. Kuo, B. Y. Chen, Y. K. Feng, Y. R. Wen, W. C. Lin, and F. H. Lin, "Toxicity Assessment of Montmorillonite as a Drug Carrier for Pharmaceutical Applications: Yeast and Rats Model," Biomed. Eng. Appl. Basis Commun., 17 (2), 12 (2005).

Lin, F. H., Y. H. Lee, C. H. Jian, J. M. Wonga, M. J. Shieha, and C. Y. Wang, “A Study of Purified Montmorillonite Intercalated with 5-Fluorouracil as Drug Carrier," Biomaterial, 23, 1981 (2002).

Lin, J.-Y., M. A. Selim, C. R. Shea, J. M. Grichnik, M. M. Omar, N. A. Monteiro-Riviere, and S. R. Pinnell, "UV Photoprotection by Combination Topical Antioxidants Vitamin C and Vitamin E," J. Am. Acad. Dermatol., 48 (6), 866 (2003).

Serpen, A. and V. Gökmen, "Reversible Degradation Kinetics of Ascorbic Acid under Reducing and Oxidizing Condition," Food Chem., 104, 721 (2007). 